\title{
Management of intraoral stents for radiotherapy during COVID-19 pandemic
}

\author{
Aljomar José Vechiato Filho ${ }^{\circledR}{ }^{1}$, Karina Morais Faria ${ }^{1}$, Maria Cecília Querido de Oliveira ${ }^{1}$, \\ Ariadne Padilha de Andrade ${ }^{1}$, Ana Carolina Prado-Ribeiro $\mathbb{1}^{1,4}$, Alan Roger Santos-Silva $\mathbb{\circledR}^{4}$, \\ Alvin G. Wee $\mathbb{1}^{5}$, Thaís Bianca Brandão ${ }^{1}{ }^{1}$ \\ ${ }^{1}$ Dental Oncology Service, Instituto do Cancer do Estado de Sao Paulo (ICESP-FMUSP) Faculdade de Medicina da Universidade de Sao Paulo, \\ Sao Paulo, Brazil \\ ${ }^{2}$ Oral Medicine Department, Hospital Sirio-Libanes, Sao Paulo, Brazil \\ ${ }^{3}$ D’Or Oncologia, São Paulo, Brazil \\ ${ }^{4}$ Oral Diagnosis Department, Piracicaba Dental School, University of Campinas (UNICAMP), Piracicaba, Sao Paulo, Brazil \\ ${ }^{5}$ Department of Restorative Sciences, School of Dentistry, University of Minnesota (UMN), Minneapolis, United States
}

\begin{abstract}
Radiotherapy is being performed in many situations as a curative approach for head and neck cancer instead of surgery due to the current novel coronavirus disease (COVID-19) pandemic. A recent publication reported that even hypofractionation was being conducted in order to reduce the daily exposure of both patients and the medical staff involved in cancer therapies. As a result, dental teams may be requested more frequently than usual to fabricate intraoral stents (IOS). Given that IOS may be a potential source of COVID-19 contagion, the main purpose of the present correspondence is to offer a guide on how health professionals may be safely presented in the room, on the management of the IOS and also how to sanitize the stents.

Key words: radiotherapy; intraoral stents; oral cancer

Rep Pract Oncol Radiother 2021;26(1):159-161
\end{abstract}

It is known that the outbreak of the novel coronavirus disease (COVID-19) has created difficult challenges for oncology clinicians in managing and treating cancer patients due to the estimated higher risk such individuals have of contracting the virus. Because of the absence of international guidelines on conducting cancer treatment during an infectious pandemic, health-care settings are postponing "elective" cancer treatments, which may be understood as newly diagnosed tumors with low proliferation rates receiving less intense chemotherapy and/or radiotherapy [1]. For example, head and neck cancer services have rescheduled surgical procedures in order to reduce the exposure of patients and the medical team to the risk of COVID-19 contagion [2]. These strategies also aim to reduce hospital length of stay, preserving clinical capacity for COVID-19 patients, which is understood to be a necessary approach given the number of patients with confirmed COVID-19 infection who will be referred and admitted to the majority of hospitals worldwide during the ongoing public health emergency.

However, a group of authors recently published an editorial letter suggesting that the postponement of cancer treatment should be avoided since such deferred care may negatively impact patients' survival. They also recommend that radiotherapy is more indicated for patients with tumors located 
in the upper aerodigestive tract if tracheostomy is required (due to the risk of COVID-19 infection, dissemination, respiratory complications, and possibly death) and also in the case of the unavailability of operating rooms, which are being used in many countries as ICUs [1]. These authors also suggest that a shorter regimen of radiation (hypofractioned) is recommended in order to reduce the daily exposure of both patients and medical staff involved in cancer therapies/treatment, without compromising control of locoregional disease progression or increasing toxicities [1]. Therefore, it can be expected that patients will probably be referred more often to the radiotherapy team. Consequently, the dental team may be requested more frequently than usual to fabricate prosthetic stents - also called intraoral stents (IOS) - for radiotherapy.

Given that dentists will probably be exposed to almost all possible routes for COVID-19 infection (aerosols, droplets, contact with contaminated materials, dental instruments and/or surfaces) [3, 4], coupled with the fact that (1) pathogenic microorganisms can be suspended in the air for long periods [4], (2) the incubation period - from 1 to 14 days, approximately - may not induce symptoms in infected patients but still turn them into a significant source of spreading, and also (3) the progressive increase in the number of infected individuals (daily updates can be verified at https://www.who. int/emergencies/diseases/novel-coronavirus-2019), it is urgent to protect patients and staff members by means of sharing clinical expertise and skills. This strategy is useful in creating guidelines for reducing exposure to the COVID-19 virus during the performance of nonelective procedures [1].

Needless to say, the current scenario does not allow the validation of all management suggestions by means of a scientific clinical trial or investigative research, whereas health professionals are demanding reliable information. Therefore, the present letter has the main goal of providing strategies based on guidelines that were developed by radiation oncologists, infectologists and dental teams of one cancer institution in Brazil (Instituto do Câncer do Estado de São Paulo - ICESP), and also on the recommendations from the World Health Organization (WHO) [5].

Figure 1 summarizes all the scripts described herein. A patient with confirmed or suspected CO-
VID-19 should be conducted to the radiotherapy equipment by two professionals who should be wearing full personal protective equipment (PPE) - disposable doctor cap, goggles, oil non-resistant air-purifying respirators with 95\% efficiency (N95) covered by a disposable American Society for Testing and Materials (ASTM) level 3 surgical mask, face shield and disposable gloves, among others. After IOS and patient positioning, one of the professionals should remove his/her PPE and perform the treatment while the second professional waits outside the radiation room, still wearing their PPE. Next, the individual wearing the PPE should remove the stent and equipment and sanitize the prosthetic device.

It is important to discuss that there is no evidence supporting the use of a particular solution for sanitizing acrylic material but Peng et al. [3] recommend the use of $0.5 \%$ hydrogen peroxide $\left(\mathrm{H}_{2} \mathrm{O}_{2}\right)$ or $\approx 70 \%$ alcohol for killing the COVID-19 virus. Therefore, the authors of the present letter suggest the use of disposable brushes with soap followed by abundant washing with water. After drying with disposable paper, $0.5 \%$ hydrogen peroxide or $\approx 70 \%$ alcohol should be sprayed onto the IOS for $1 \mathrm{~min}$ and cleaned. Finally, the IOS should be stored in a proper box that allows airflow.

One of the goals of the present letter is to avoid the situation radiotherapists not requesting IOS fabrication during the COVID-19 pandemic because of the risk of contagion, as discussed above. In this context, it is important to consider that Chaves et al. [1] suggested that multidisciplinary support must be maintained during patient's treatment and that IOS may reduce the incidence and severity of an important acute toxicity related to radiation: oral mucositis. It is known that this complication can negatively impact patients' outcomes (such as: pain, inability to eat with requirement for feeding tubes, and weight loss), increasing resource usage (drugs, hospitalization, for example) [6], and consequently, may facilitate infection with COVID-19 and mortality.

\section{Conflict of interest}

The authors declare that they have no conflict of interests or personal relationships that could lead this publication to bias.

\section{Funding}

The authors declare that no financial support was used for the conduction of this publication. 


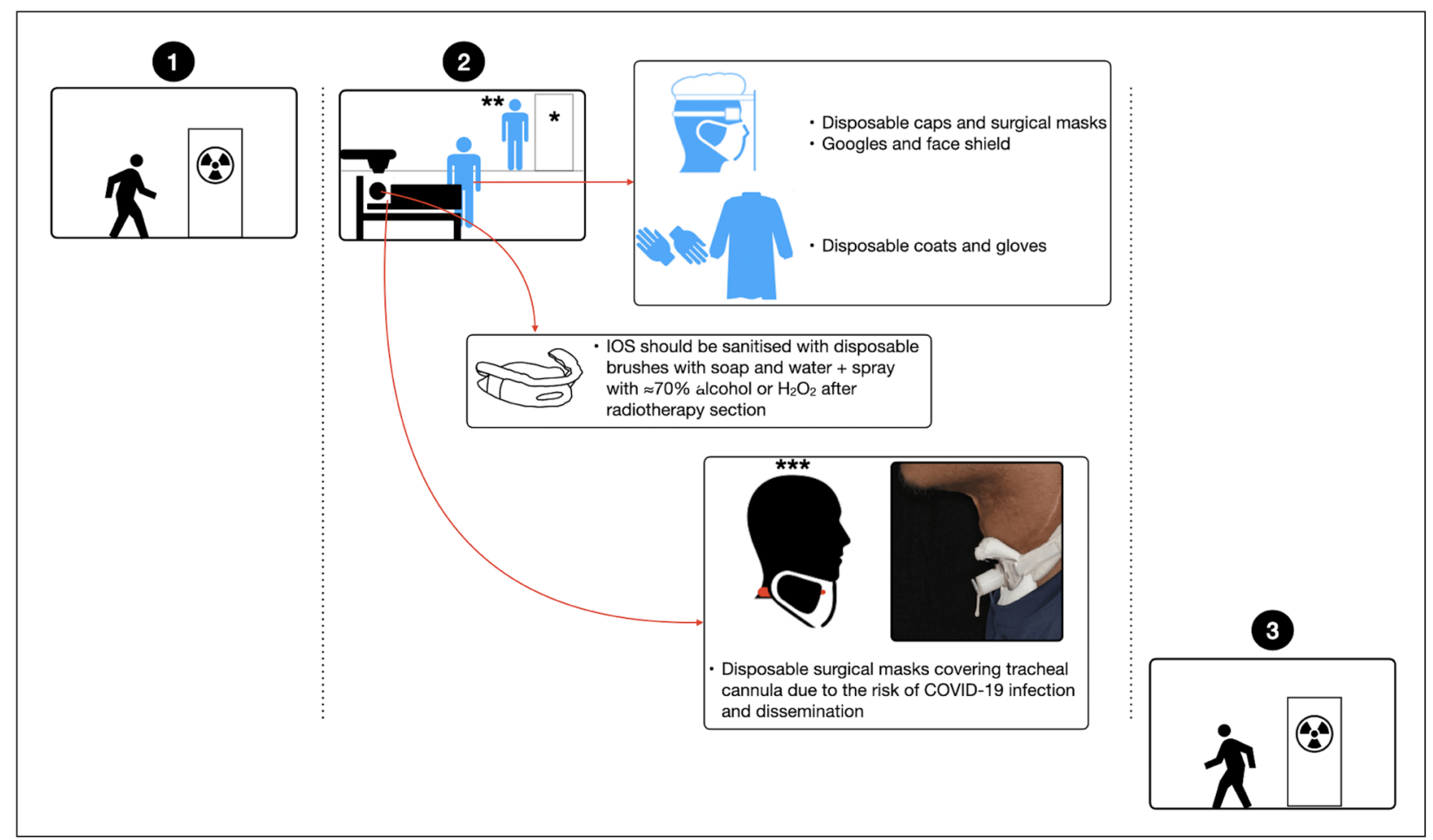

Figure 1. Scheme summarizing all the information necessary for the safe manipulation of intraoral stents during the radiation treatment. 1 - patient arrives in the radiotherapy room; 2 - during radiotherapy session: ${ }^{*}$ door of the hospital room should be closed with low pressure inside in cases of suspected or confirmed COVID-19; ** one radiation technician should remove disposable protective equipment and wait outside the hospital room during the radiation treatment; ${ }^{* * *}$ Patients with tracheostomy should have their tracheal cannula covered with a disposable surgical mask because this could become a relevant infection route if the individual coughs, secreting fluids, during the session. It is also very important that professionals sanitize their hands with water and soap or alcohol gel before the patient examination, before dental procedures, after touching the patient, after touching equipment without disinfection, and after touching the oral mucosa or body fluids [3].3 - patient leaves the radiotherapy room

\section{References}

1. Chaves AL, Castro AF, Marta GN, et al. Emergency changes in international guidelines on treatment for head and neck cancer patients during the COVID-19 pandemic. Oral Oncol. 2020; 107: 104734, doi: 10.1016/j.oraloncology.2020.104734, indexed in Pubmed: 32353793.

2. Yang Y, Soh HY, Cai ZG, et al. Experience of Diagnosing and Managing Patients in Oral Maxillofacial Surgery during the Prevention and Control Period of the New Coronavirus Pneumonia. Chin J Dent Res. 2020; 23(1): 57-62, doi: 10.3290/j.cjdr.a44339, indexed in Pubmed: 32232230.

3. Peng X, Xu X, Li Y, et al. Transmission routes of 2019$\mathrm{nCoV}$ and controls in dental practice. Int J Oral Sci. 2020; 12(1): 9, doi: 10.1038/s41368-020-0075-9, indexed in Pubmed: 32127517.
4. Kampf G, Todt D, Pfaender S, et al. Persistence of coronaviruses on inanimate surfaces and their inactivation with biocidal agents. J Hosp Infect. 2020; 104(3): 246-251, doi: 10.1016/j.jhin.2020.01.022, indexed in Pubmed: 32035997.

5. World Health Organization (WHO). Infection prevention and control during healthcare when COVID-19 is suspected. Interim guidance. https://www.who.int/ publications-detail/infection-prevention-and-controlduring-health-care-when-novel-coronavirus-(ncov)infection-is-suspected-20200125 (09 April 2020).

6. Elting LS, Cooksley CD, Chambers MS, et al. Risk, outcomes, and costs of radiation-induced oral mucositis among patients with head-and-neck malignancies. Int J Radiat Oncol Biol Phys. 2007; 68(4): 1110-1120, doi: 10.1016/j. ijrobp.2007.01.053, indexed in Pubmed: 17398022. 